\title{
ORIGINAL
}

\section{CONSUMO EPISÓDICO EXCESIVO DE ALCOHOL EN ADOLESCENTES: SU ASOCIACIÓN CON LOS ESTADOS DE ÁNIMO NEGATIVOS Y LOS FACTORES FAMILIARES (*)}

\author{
Angel Martínez-Hernáez (1,2), Marga Marí-Klose (3,4), Albert Julià (4), Sandra Escapa $(3,4)$ y Pau \\ Marí-Klose (3,5).
}

(1)Universitat Rovira i Virgili

(2)Universidade Federal de Santa Catarina

(3)Universitat de Barcelona

(4)Institut d'Infància i Món Urbà

(5)Instituto de Políticas y Bienes Públicos. Consejo Superior de Investigaciones Científicas (CSIC)

(*) Esta investigación se ha realizado en el marco del proyecto "The emotional distress of adolescents: lyfestyles, mental health, and lay strategies used in managing adversity", código 090730/31, financiado por Fundació la Marató de TV3. También se ha recibido financiación del proyecto "Los malestares de los adolescentes" (MICINN-CSO2009-08432). No existe conflicto de interés.

\section{RESUMEN}

Fundamentos: El consumo episódico excesivo de alcohol (CEEA) es una práctica extendida en la población adolescente con graves riesgos para la salud, incluyendo el abuso/dependencia en la edad adulta. El objetivo de este artículo es analizar su asociación con los estados de ánimo negativos y algunas variables familiares entre los adolescentes catalanes.

Métodos: Estudio transversal de una muestra representativa de adolescentes (edad 14-18 años) de Cataluña (Segunda oleada del Panel de Familias e Infancia) (2006-2010). Se realizan modelos de regresión logística para mujeres $(\mathrm{n}=1.459)$ y hombres $(\mathrm{n}=1.105)$ para estimar $\mathrm{si}$ los estados de ánimo negativos auto-percibidos (tristeza, presión parental, etc.) están asociados con el CEEA, capturado como haberse emborrachado por lo menos dos veces al mes durante el último año. Se estima en qué medida esos efectos son atribuibles a algunos factores familiares.

Resultados: El sentimiento crónico de tristeza está asociado con el CEEA entre los varones (OR 2,7), al igual que sentirse presionado/a por los progenitores en ambos sexos (OR 1,8 hombres y OR 2,1 mujeres). Las mujeres de rentas medio-bajas y altas son más proclives al CEEA (OR 1,6 y OR 1,7 respectivamente). La existencia de progenitores de origen inmigrante $(\mathrm{OR} 0,4)$ y la fijación parental de un horario de retorno a casa los fines de semana (OR 0,6) presentan una asociación negativa con el CEEA entre las mujeres.

Conclusiones: Los estados de ánimo negativos (EAN) están asociados al CEEA. Los efectos de los factores socio-económicos y familiares sobre el CEEA son más relevantes en mujeres que en varones

Palabras clave: Consumo de bebidas alcohólicas, Adolescente, Afecto, Relaciones familiares.

Correspondencia

Angel Martínez-Hernáez

Departamento de Antropología

Universitat Rovira i Virgili

Avinguda Catalunya, 35

43002 Tarragona

angel.martinez@urv.cat; amhernaez@gmail.com

\section{ABSTRACT}

\section{Heavy Episodic Drinking Among Adolescents: The Association with Negative Mood States and Family Factors}

Background: Heavy episodic drinking is widespread among adolescents, with serious health risks, including abuse / dependence in adulthood. The aim of this paper is to analyze the influence of negative mood states and some family variables on this type of drinking behavior among Catalan adolescents.

Methods: Cross sectional study of a representative sample of adolescents (age 14-18 years) from Catalonia (Second Wave, Panel of Families and Children) (2006-2010). Separate logistic regression models are run for women $(\mathrm{n}=1,459)$ and men $(\mathrm{n}=1,105)$ to assess whether negative mood states (self-perceived) are associated with heavy episodic drinking measured as binge drinking at least twice a month or more in the last year. It is estimated to what extent these effects are attributable to familial factors.

Results: Feelings of sadness are associated with binge drinking among male adolescents (OR 2.7). Feeling pressured by parents keeps also a positive association with binge drinking among both sexes (OR 1.8 for males and OR 2.1 for women). Women from low-middle and high income groups are more likely to engage in binge drinking (OR 1.6 and OR, 1.7 respectively). Migrant family background (OR 0.4 ) and parental control of arrival home on weekend (OR 0.6) are negatively related to binge drinking among female adolescents.

Conclusions: Negative mood states are associated with heavy episodic drinking. Socio-economic and family factors have stronger effect on women than on men. ions.

Keywords: Alcohol Drinking, Adolescent, Affect, Family Rela- 


\section{INTRODUCCIÓN}

El Consumo Episódico Excesivo de Alcohol (CEEA), también conocido en español como Consumo Intensivo de Alcohol (CIA) y en el panorama internacional como binge drinking o heavy episodic drinking, es una práctica común entre los adolescentes caracterizada por la ingesta de grandes cantidades de alcohol en un único episodio: usualmente 5 bebidas o más para los varones y 4 o más para las mujeres, con $80 \mathrm{mg} / \mathrm{dl}$ de concentración en sangre o superior (hombres) y $60 \mathrm{mg} / \mathrm{dl}$ (mujeres) ${ }^{1,2}$. El riesgo para la salud del CEEA ha sido descrito extensamente e incluye factores como la mayor probabilidad de ingresos hospitalarios, suicidio, hipertensión, pancreatitis, patología hepática, violencia y victimización sexual, accidentalidad viaria, policonsumo de substancias, bajo rendimiento escolar, problemas neurocognitivos y trastornos de la conducta alimentaria, entre otros ${ }^{1-}$ 7. De hecho, el consumo de alcohol es una de las principales causas de mortalidad entre los jóvenes ${ }^{8}$. Adicionalmente, el CEEA está asociado con la existencia de consumo de riesgo y de abuso/dependencia en la edad adulta ${ }^{9,10}$ y con trastornos y síntomas del espectro depresivo/ansioso ${ }^{11,12}$.

La literatura indica la presencia de diferentes patrones sociales y culturales de CEEA. Así, mientras que en Holanda se calcula que un $75 \%$ de los adolescentes de 17 años lo practica al menos una vez al mes, este porcentaje se reduce aproximadamente al $50 \%$ en otros países europeos ${ }^{13}$. Según la Encuesta Domiciliaria sobre Alcohol y Drogas en España (EDADES) de $2009^{14}$, la prevalencia de CEEA mensual es de $28,4 \%$ para los varones de 15 a 24 años y de $17,9 \%$ para las mujeres. En la Encuesta
Estatal sobre Uso de Drogas en Estudiantes de Enseñanzas Secundarias (ESTUDES) de $2008^{15}$, las prevalencias mensuales para las edades de $14,15,16$, 17 y 18 años son de $13,4 \%, 24,2 \%$, $31,7 \%, 39,0 \%, 45,2 \%$ respectivamente. Asimismo, una investigación longitudinal reciente en población universitaria gallega ${ }^{16}$ muestra que el CEEA disminuye con el tránsito a la juventud mientras que aumenta ostensiblemente el consumo de riesgo. Estos últimos datos son congruentes con los resultados de otros países, que señalan una mayor prevalencia entre los más jóvenes y una disminución asociada al aumento de la edad. Se estima que la prevalencia de CEEA en población adolescente y joven es el doble a la de la población adulta ${ }^{17}$.

Algunos estudios indican que entre el 40 y el $60 \%$ de la probabilidad de desarrollar un consumo de riesgo de alcohol y trastorno por abuso/dependencia puede ser atribuido a factores genéti$\cos ^{18-21}$.Sin embargo, los determinantes genéticos de una práctica enraizada socialmente como el CEEA, difícilmente pueden dar cuenta de toda su complejidad. Es cierto que hay factores que invitan a pensar en la importancia del componente biológico. Un estudio reciente de Holstein et $\mathrm{al}^{20}$. en ratones apunta que los individuos jóvenes podrían tener menor sentido de aversión al alcohol, lo cual explicaría la mayor prevalencia de binges en adolescentes humanos. También se ha observado que los jóvenes con la versión corta del gen transportador serotoninérgico (5-HTT) tienen un mayor consumo de alcohol por episodio, beben más a menudo para embriagarse y practican el CEEA de forma más frecuente que los sujetos sin esta variante genética ${ }^{21}$. Sin embargo, el papel de los factores sociales y psicosociales no debe minusvalorarse. La pro- 
clividad del grupo de iguales ${ }^{22}$, la ausencia de políticas que dificulten el acceso a las bebidas alcohólicas ${ }^{23}$ y las diferencias culturales y religiosas ${ }^{22}$ son factores de riesgo conocidos. Adicionalmente, los factores familiares presentan una asociación con el CEEA y el consumo de alcohol en general, aunque con resultados dispares. Por un lado, la tolerancia parental al uso/consumo de alcohol, el consumo por parte de los progenitores, la existencia de pocos lazos familiares, la ausencia de control parental de las actividades de los adolescentes o de vínculos de comunicación, afecto y apoyo son factores de riesgo robustos según diversas investigaciones ${ }^{25-28}$. Por otro lado, sin embargo, el estatus socio-económico y la estructura de las familias muestran un efecto muy limitado ${ }^{29}$.

Tanto el entorno familiar como los estados de ánimo negativos de los adolescentes son influencias conocidas sobre el CEEA que, sin embargo, no se han investigado conjuntamente en nuestro contexto social. Existen estudios que analizan la relación entre el CEEA y los factores familiares ${ }^{29-31}$. También que vinculan los estados de ánimo negativos con este tipo de consumo ${ }^{32}$. Pero no investigaciones que determinen la asociación entre CEEA y estados de ánimo de los adolescentes, controlando los efectos atribuibles a factores relacionados con el contexto familiar. Y ello a pesar de que una de las hipótesis más consolidadas sobre el consumo de alcohol y de substancias en general es la self-medication theory: los jóvenes beberían intensivamente para mitigar los estados de ánimo negativos de tipo depresivo y ansioso ${ }^{11}$. Con todo, la relación entre malestar emocional y CEEA parece ser bidireccional: los síntomas depresivos predicen el CEEA y este último puede ser un factor de riesgo de depresión y estados de ánimo negativos como consecuencia de los efectos de la intoxicación etílica en los niveles serotoninérgicos ${ }^{33}$. En cualquier caso, parece acertado afirmar que generalmente se produce un concurrencia malestar emocional y CEEA y, en las formas más severas, una comorbilidad depresión y abuso/dependencia del alcohol ${ }^{34}$.

El objetivo de este artículo es analizar la asociación entre los estados de ánimo negativos (EAN) autopercibidos y CEEA (auto-informado) entre los adolescentes catalanes, controlando algunos de los principales factores sociofamiliares (estructura del hogar, nivel de renta, factor migratorio, comunicación y control parental de los horarios de los adolescentes) que influyen sobre esta forma de consumo.

\section{SUJETOS Y MÉTODOS}

\section{Diseño, población y recolección de} datos. Los datos de este estudio proceden del Panel de Familias e Infancia $(\mathrm{PFI})^{35}$, una encuesta longitudinal diseñada y realizada por el Instituto de Infancia y Mundo Urbano (CIIMU) que se aplicó durante cuatro años consecutivos desde 2006. El PFI recopiló información sobre consumo de substancias psicoactivas, EAN y una amplia diversidad de factores sociales, escolares y familiares. Para seleccionar a los participantes, el Panel de Familias e Infancia utilizó un procedimiento de muestreo polietápico estratificado. En primer lugar se seleccionó una muestra de 70 centros de Educación Secundaria Obligatoria en función de su localización geográfica en clusters territoriales definidos previamente por características socio-económicas (obtenidos a partir del Censo del año 2001), respetando asimismo la representación proporcional en cuanto a la titularidad de centros. En 
segundo lugar se seleccionó a los alumnos de cada centro que cursaban entre primero y cuarto de la ESO. La muestra representativa inicial contaba con 3.004 adolescentes residentes en Cataluña. Los datos fueron obtenidos mediante encuesta auto-administrada a los adolescentes en sus centros escolares y en su domicilio se entrevistó al progenitor/tutor que había firmado el consentimiento (en el 79,4\% de los casos era la madre). La edad media de las madres que participaron en la encuesta fue de 43,9 años y la de los padres de 46,4. La distribución del nivel de estudios de madres y padres fue similar: el 25,6\% de madres y un $23,9 \%$ de padres tenían estudios universitarios; el $40 \%$ de las madres y el $38,6 \%$ de padres tenía estudios secundarios y $34,4 \%$ de las primeras frente al $35,7 \%$ de los segundos tenía estudios primarios. En el momento de la entrevista trabajaban el $81,1 \%$ de las madres y el $97,3 \%$ de los padres.

Las encuestas a los adolescentes se realizaron entre abril y junio de cada curso escolar (2006, 2007 y 2008) y a los progenitores entre octubre y diciembre de 2006. En este artículo analizamos los datos procedentes de la entrevista a los adolescentes en la segunda oleada (2007), ya que ésta incluye una batería de preguntas sobre EAN y relaciones intergeneracionales, mientras que los datos correspondientes a la estructura familiar, renta y origen inmigrante proceden de la entrevista realizada a los progenitores (2006).

Los adolescentes que respondieron al cuestionario en 2007 tenían entre 14 y 17-18 años. La muestra mantuvo 2.850 sujetos que habían participado en la primera oleada. De la muestra se eliminaron $285(10 \%)$ cuestionarios por información incompleta sobre las variables de análisis, resultando una muestra final de 2.564 casos (1.459 mujeres y 1.105 varones).

\section{Definición de las variables.}

Consumo episódico excesivo de alcohol. La pregunta inicial fue si había bebido alguna vez cerveza, vino u otras bebidas alcohólicas cuando no estaba con sus padres u otras personas adultas de la familia. En caso de respuesta positiva, el sujeto debía responder sobre las veces que se emborrachó o se puso muy contento con bebidas alcohólicas en el último año. Las respuestas se codificaron en una variable dicotómica: a) Casi cada día $=1$, b) Tres a cuatro veces por semana $=1, \mathrm{c})$ Dos o tres veces al mes $=1, d$ ) Una vez al mes o menos (de tres a doce veces en el último año) $=0$, e) Una o dos veces al año $=0, f$ ) Nunca $=0$. Los entrevistados que en la pregunta inicial respondieron que no habían consumido nunca alcohol cuando no estaban en presencia de padres u otros adultos fueron codificados también como 0 .

Estados de ánimo negativos. La pregunta formulada fue: Todo el mundo pasa por diferentes estados de ánimo ¿con qué frecuencia te sientes triste, nervioso, aburrido, cansado, solo, demasiado ocupado para hacerlo todo, presionado por tus padres? Las frecuencias para cada uno de los ítems fueron recodificadas en variables dicotómicas: a) nunca o casi nunca $=0$, b) a veces $=0$, c) a menudo $=1$.

Nivel de renta familiar. Se elaboraron cuatro variables dicotómicas correspondientes con cuatro cuartiles de renta anual de los hogares. El cuartil 1 pertenece a las rentas más bajas y el cuartil 4 a las más altas. 
Estructura familiar. Se construyeron tres variables dicotómicas para cada tipo de hogar: monoparental, biparental, reconstituido (formado por un progenitor y una nueva pareja).

Origen inmigrante. Se elaboró una variable dicotómica donde $1=$ ambos progenitores son de origen extranjero, y $0=$ progenitores nacidos en España.

Comunicación intergeneracional. La pregunta realizada fue: "En el último mes ¿con qué frecuencia has hablado con tu madre y con tu padre de los siguientes temas: la escuela y los profesores, lo que haces con tus amigos, cosas que pasan en el mundo o salen en las noticias, deportes, y chicos/chicas que te gustan? Para cada ítem se ofrecieron 6 opciones de respuesta: 1) nunca, 2) una o dos veces al mes, 3) una vez a la semana, 4) dos o tres veces por semana, 5) casi todos los días, 6) todos los días. Con estos ítems se construyó un índice aditivo en el que los valores más altos correspondieron a los niveles de comunicación más intensos.

Norma sobre horarios del fin semana. La pregunta realizada fue "por favor, dinos si en tu casa te dicen la hora a la que tienes que llegar todos los fines de semana". La respuesta es dicotómica. "No" es el valor de referencia utilizado.

Análisis estadístico. Se calcularon las prevalencias de consumo por sexo y edad. Posteriormente se efectuaron modelos de regresión logística independientes para hombres y mujeres. Los EAN estadísticamente significativos en el análisis bivariado fueron incluidos en los modelos (sentimiento de tristeza y sentirse presionados por los progenitores) con el propósito de observar si los afectados tenían un mayor riesgo de CEEA. Sucesivamente se incorporaron las variables familiares para analizar cómo afecta su introducción al efecto de los EAN (aunque aquí se opta por no reproducir todos los modelos). En primer lugar se incluye la edad y el EAN (tristeza o sentirse presionado). A continuación se incorporan la estructura familiar, el nivel de ingresos del hogar, el origen de los progenitores, la calidad de la comunicación intergeneracional y la norma sobre horarios. Siguiendo los procedimientos habituales en Ciencias Sociales, se identificaron cuatro niveles de significación estadística $(\dagger \mathrm{p}<0,1$ : $* \mathrm{p}<0,05 ; * * \mathrm{p}<0,01 ; * * * \mathrm{p}<0,001)$. Los análisis se realizaron con el paquete estadístico SPSS 16.0.

\section{RESULTADOS}

En la tabla 1 se puede observar la distribución según sexo y edad de consumo de alcohol y la frecuencia en que se ha producido de CEEA en año 2007 entre adolescentes. E1 64,6\% consumió alcohol alguna vez sin la presencia de sus padres $u$ otros adultos de la familia. Con la edad, la proporción de adolescentes que probaron el alcohol aumentó. En cuanto a las formas más intensivas de consumo, el $32,4 \%$ reconocíó haberlo hecho una o dos veces en el último año.

La prevalencia de CEEA aumentó con la edad. Del conjunto de estados de ánimo analizados, el análisis bivariado (tabla 2) nos indicó que los estados crónicos de nerviosismo, aburrimiento, cansancio, soledad o sentirse demasiado ocupado para hacerlo todo no mostraban una asociación estadísticamente significativa con el CEEA. Sólo fueron significativos la tristeza crónica para el caso de los adolescentes (OR 2,7, IC $95 \% 1,4-5,0)$ y sentirse frecuentemente presionados por los padres para ambos sexos (OR 2,1, IC 95\% 1,4-3,2 para 
Tabla 1

Prevalencias de consumo de alcohol en jóvenes de 14-18 años, según sexo y edad

\begin{tabular}{|l|c|c|c|c|c|}
\hline & $\begin{array}{c}\text { Alguna vez } \\
\text { en la vida }\end{array}$ & $\begin{array}{c}\text { Un o dos veces } \\
\text { en el último año }\end{array}$ & $\begin{array}{c}\text { De tres a doce } \\
\text { veces en el último } \\
\text { año }\end{array}$ & $\begin{array}{c}\text { Dos o tres veces } \\
\text { al mes }\end{array}$ & $\begin{array}{c}\text { Una o más veces a } \\
\text { la semana }\end{array}$ \\
\hline Total & $64,60 \%$ & $32,40 \%$ & $16,80 \%$ & $13,20 \%$ & $5,30 \%$ \\
\hline Sexo & & & & & \\
\hline Mujeres & $66,90 \%$ & $32,60 \%$ & $17,90 \%$ & $14,10 \%$ & $4,10 \%$ \\
\hline Hombres & $61,50 \%$ & $32,10 \%$ & $15,30 \%$ & $11,90 \%$ & $6,90 \%$ \\
\hline Edad & & & & & \\
\hline 14 años & $39,70 \%$ & $37,90 \%$ & $9,30 \%$ & $4,80 \%$ & $1,40 \%$ \\
\hline 15 años & $60,00 \%$ & $34,30 \%$ & $11,30 \%$ & $10,50 \%$ & $3,90 \%$ \\
\hline 16 años & $77,60 \%$ & $32,80 \%$ & $19,20 \%$ & $12,90 \%$ & $5,30 \%$ \\
\hline 17 y 18 años & $86,80 \%$ & $26,90 \%$ & $23,20 \%$ & $21,00 \%$ & $8,70 \%$ \\
\hline
\end{tabular}

mujeres y OR 1,8, IC 95\% 1,1-3,0 para hombres)

La estructura de la familia (biparentalidad, monoparentalidad, familia reconstituida) no estuvo asociada con el CEEA, pero sí la pertenencia al cuartil de renta más alta en el caso de las mujeres (OR 1,7, IC 95\% 1,0 - 2,8). Entre ellas, también se observó una asociación negativa del CEEA con familia de progenitores extranjeros (OR 0,4, IC $95 \% 0,2-1,0)$ y con la existencia de control parental en el horario de retorno a casa los fines de semana (OR 0,6, IC $95 \% 0,4-0,9)$.

Buena parte de las relaciones bivariadas detectadas seguían siendo estadísticamente significativas cuando se controlaron los efectos en una regresión logística multivariante. En cuanto al sentimiento crónico de tristeza, la tabla 3 muestra los resultados tras incluir las variables familiares en el modelo en el caso de los varones adolescentes. A iguales condiciones de edad, estructura familiar, renta, origen autóctono/inmigrante de los progenitores, sentirse presionado y comunicación con el padre y con la madre, se observó que este estado de ánimo mantuvo una relación estadísticamente significativa con el CEEA (OR 2,8 IC $95 \%$ 1,4 - 5,8). Asimismo, controlando por las mismas variables, sentirse presionado por los progenitores estuvo asociado tanto entre los adolescentes (OR 1,6 IC 95\% 0,9 - 2,9) (tabla 3) como entre las jóvenes (OR 2,3 IC $95 \% 1,3$ - 3,4) (tabla 4). Entre estas últimas, se advierte una asociación estadísticamente significativa del CEEA con los cuartiles de renta 2 (OR 1,9 IC $95 \% 1,1-3,3$ ) y 4 (OR 2,0 IC $95 \% 1,1-3,4)$ y con el nivel de comunicación con el padre (OR 0,9 IC 95\% 0,9-1,0). 


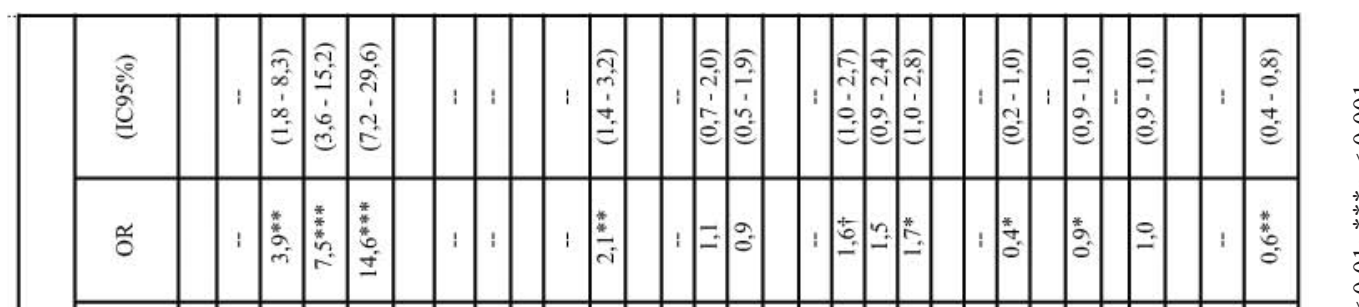

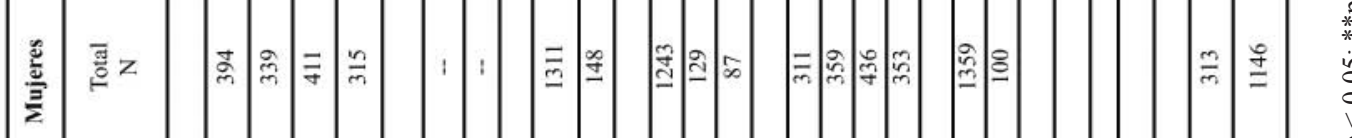

近可

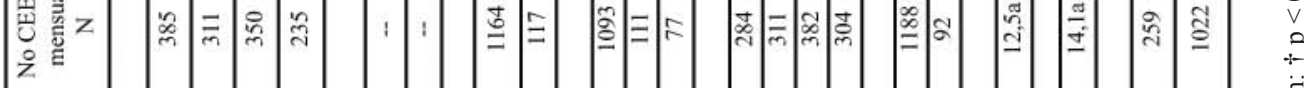

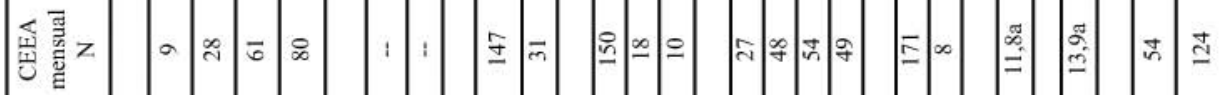

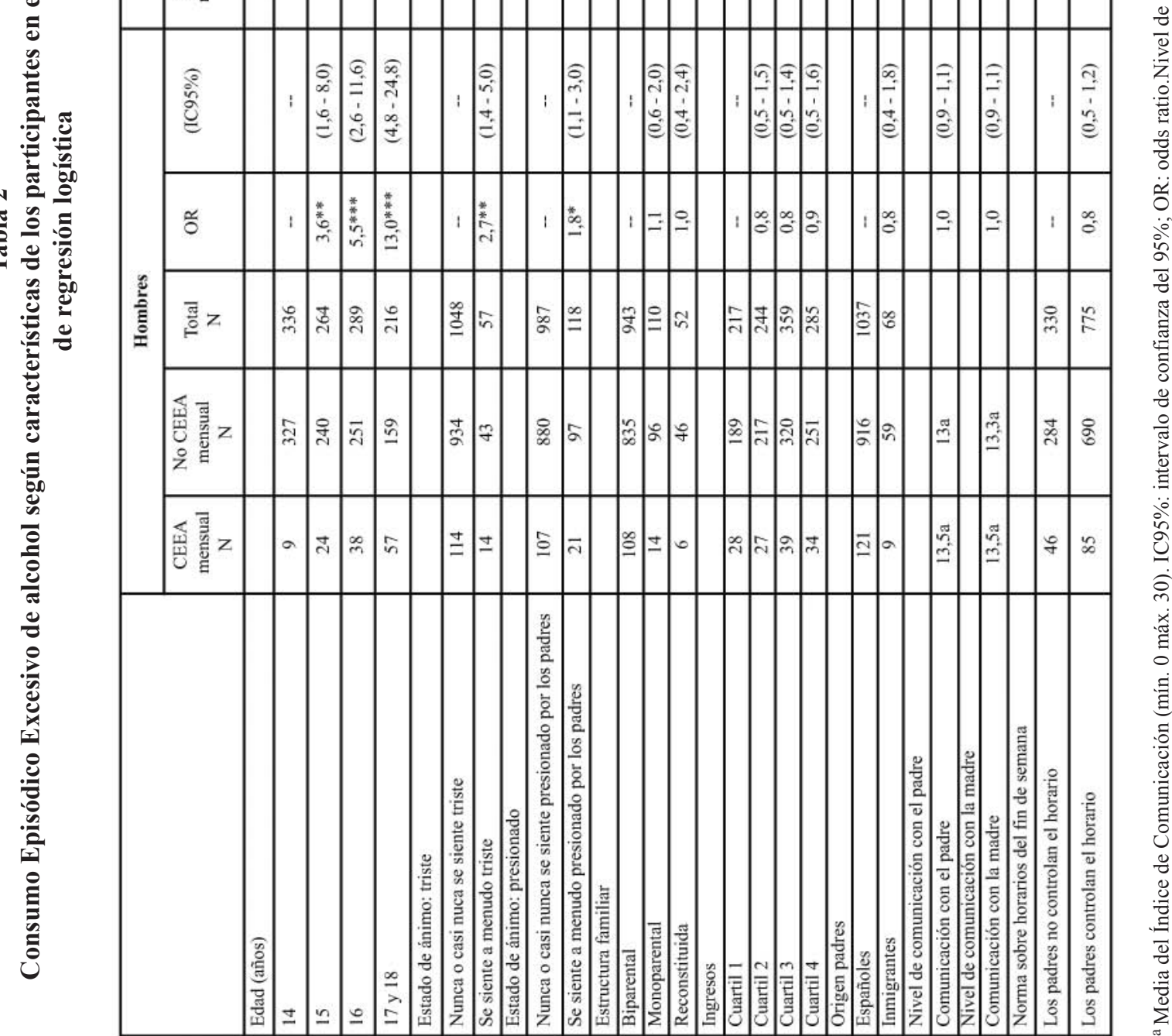




\section{Tabla 3}

Modelo de regresión logística. Análisis de los efectos de sentirse triste y presionado sobre el Consumo Episódico Excesivo de Alcohol, controlando factores del entorno familiar (Hombres)

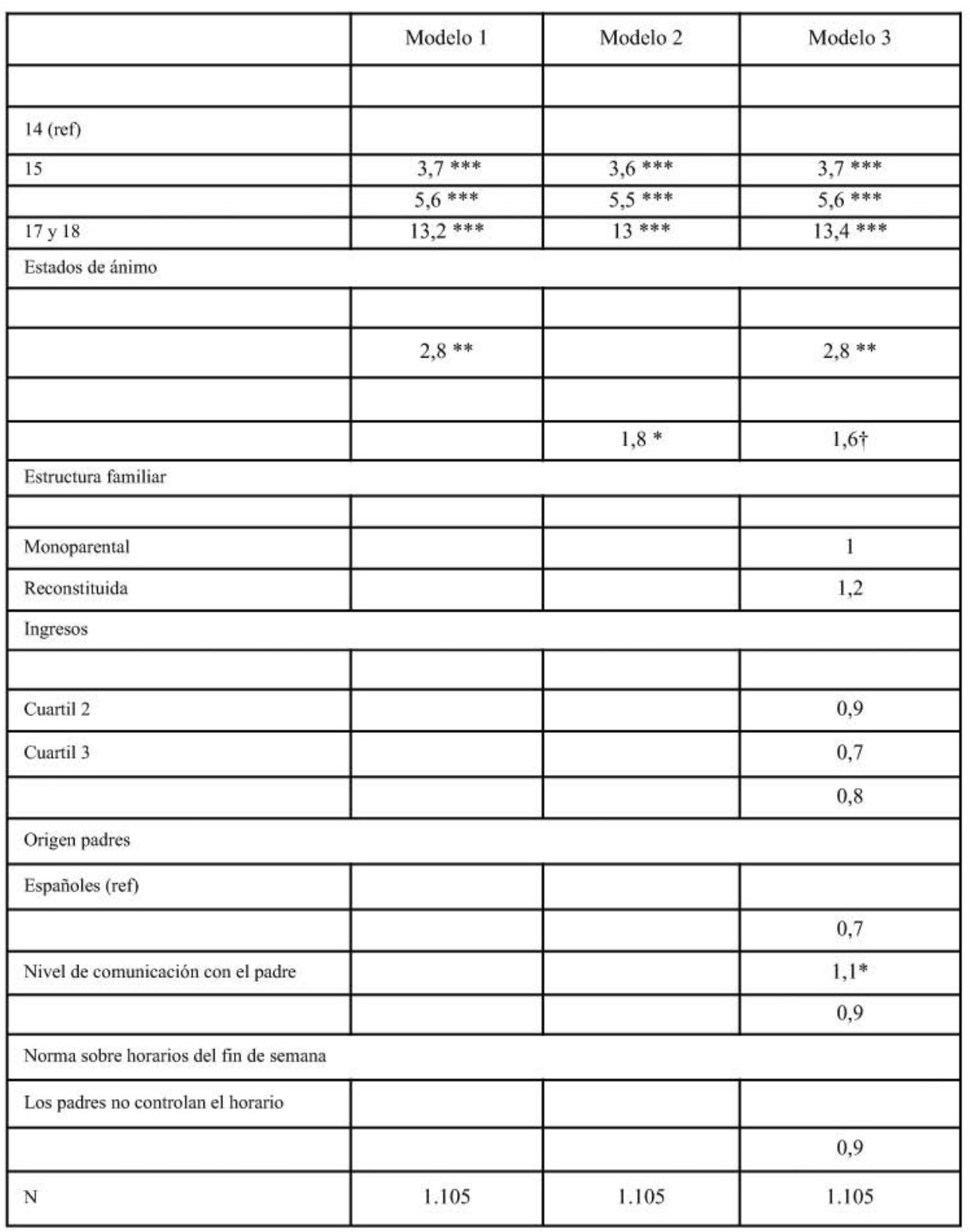

Nivel de significación: † $\mathrm{p}<0,1 ;{ }^{*} \mathrm{p}<0,05 ;{ }^{* *} \mathrm{p}<0,01 ; * * * \mathrm{p}<0,001$. 
Tabla 4

Modelo de regresión logística. Análisis de los efectos de sentirse presionado sobre el Consumo Episódico Excesivo de Alcohol, controlando factores del entorno familiar (Mujeres)

\begin{tabular}{|c|c|c|}
\hline Variables de control: & Modelo 1 & Modelo 2 \\
\hline \multicolumn{3}{|l|}{ Edad (años) } \\
\hline \multicolumn{3}{|l|}{14 (ref) } \\
\hline 15 & $3,8 * * *$ & $4 * * *$ \\
\hline 16 & 7,4 *** & $7,8 * * *$ \\
\hline 17 y 18 & 14,7 & $14,6 * * *$ \\
\hline \multicolumn{3}{|l|}{ Estado de ánimo } \\
\hline \multicolumn{3}{|c|}{ Nunca o casi nunca se siente presionado por los padres (ref) } \\
\hline Se siente a menudo presionado por los padres & $2,1 * *$ & $2,3^{* *}$ \\
\hline \multicolumn{3}{|l|}{ Estructura familiar } \\
\hline \multicolumn{3}{|l|}{ Biparental (ref) } \\
\hline Monoparental & & 1,1 \\
\hline Reconstituida & & 1,2 \\
\hline \multicolumn{3}{|l|}{ Ingresos } \\
\hline \multicolumn{3}{|l|}{ Cuartil 1 (ref) } \\
\hline Cuartil 2 & & $1,9 *$ \\
\hline Cuartil 3 & & $1,7 \dagger$ \\
\hline Cuartil 4 & & $2 *$ \\
\hline \multicolumn{3}{|l|}{ Origen padres } \\
\hline \multicolumn{3}{|l|}{ Españoles (ref) } \\
\hline Inmigrantes & & $0,3 *$ \\
\hline \multicolumn{3}{|l|}{ Nivel de comunicación con el padre } \\
\hline Comunicación con el padre & & $0,9^{* *}$ \\
\hline \multicolumn{3}{|l|}{ Nivel de comunicación con la madre } \\
\hline Comunicación con la madre & & 1 \\
\hline \multicolumn{3}{|l|}{ Norma sobre horarios del fin de semana } \\
\hline \multicolumn{3}{|l|}{ Los padres no controlan el horario } \\
\hline Los padres controlan el horario & & $0,6^{* *}$ \\
\hline $\mathrm{N}$ & 1.459 & 1.459 \\
\hline
\end{tabular}

Nivel de significación: †p $<0,1 ;{ }^{*} \mathrm{p}<0,05 ; *{ }^{*} \mathrm{p}<0,01 ; * *{ }^{*} \mathrm{p}<0,001$ 


\section{DISCUSIÓN}

En nuestro estudio se observa una asociación robusta entre sentimiento crónico de tristeza y CEEA en los adolescentes varones pero no en las mujeres. Sentirse presionados por los progenitores, en cambio, aparece asociado en ambos sexos para este tipo de consumo de alcohol. Las mujeres constituyen, aparentemente, un grupo más permeable a la influencia de los factores socio-económicos y familiares. Entre ellas, la mayor frecuencia de CEEA se produce principalmente en el grupo de mayor renta, en comparación con las rentas más bajas. También entre ellas el origen inmigrante de la familia, la existencia de un control parental de la hora de llegada a casa los fines de semana y el grado de comunicación que mantienen con el padre tienden a reducir la probabilidad de que se produzcan atracones o binges de alcohol.

No obstante, estos resultados deben valorarse a la luz de las limitaciones que tiene esta investigación. La primera de ellas es que la información sobre el CEEA refiere a la autopercepción por parte de los adolescentes y no a su medición objetiva o a la aplicación de una escala validada en nuestro entorno. Una situación semejante se desprende de los datos obtenidos sobre los EAN. Por otro lado, la información sobre variables familiares proviene de oleadas diferentes de una encuesta longitudinal, aunque como el lapso temporal es de apenas seis meses de media, resulta poco probable que se hayan generado modificaciones significativas en las unidades domésticas. Otra limitación tiene que ver con los condicionantes derivados del carácter transversal de la investigación, que no permite establecer un análisis más en profundidad del impacto de los EAN y la dirección de las asociaciones detectadas. Asimismo, no dispusimos de informa- ción sobre variables que potencialmente podrían arrojar más luz sobre los determinantes del CEEA, como el consumo de alcohol en la unidad doméstica por parte de los progenitores. Sin duda, esta información habría permitido analizar más en detalle algunas cuestiones, como si el mayor riesgo de CEEA entre las adolescentes de familias con mayor renta estuviera asociado también a un mayor consumo de bebidas alcohólicas en este tipo de hogares. Por último, cabe señalar que 420 individuos de la muestra inicial $(13,9 \%)$ no figuran en los modelos estadísticos presentados, ya sea porque no participaron en la segunda ola del Panel realizada en 2007, o porque proporcionaron información incompleta en sus cuestionarios. Aproximadamente la mitad de missings correspondió a adolescentes que no contestaron a la pregunta sobre nivel de comunicación con el padre (por defunción del padre, no reconocimiento del hijo, divorcio o separación). No hay razones, por tanto, para pensar que la exclusión de estos casos introduzca sesgos significativos. Los casos no incluidos en los análisis presentan valores y distribuciones similares al resto de la muestra en las variables sociodemográficas más importantes (edad de los adolescentes, ingresos del hogar, y nivel educativo de los padres).

Las prevalencias de CEEA de nuestro estudio presentan algunas diferencias con las que aparecen en otros, como en la Encuesta Domiciliaria sobre Alcohol y Drogas en España (EDADES) de $2009^{14}$ y en la Encuesta Estatal sobre Uso de Drogas en Estudiantes de Enseñanzas Secundarias (ESTUDES) de $2008^{15}$. La disparidad de las cifras obtenidas debe atribuirse fundamentalmente a distintas formas de medir el CEEA, tal como ya se ha puesto en evidencia en la literatura ${ }^{36}$. Nuestro indicador captura un consumo de alcohol más intensivo (emborracharse 
dos o más veces al mes) que el utilizado por EDADES y ETUDES ${ }^{14,15}$ (una vez al mes). Además, en nuestro caso hemos agrupado a los jóvenes con edades de 17 y 18 años porque la proporción de los últimos era muy baja (se trata, en nuestra muestra, de adolescentes que han repetido curso alguna vez). Representan un $10 \%$ de esta categoría de edad (17 años $n=478 ; 18$ años $n=53$ ), por lo que la prevalencia de consumo en este grupo de edad se ajusta más a la de los que tienen 17 años en otros estudios.

En nuestra investigación no encontramos una asociación significativa entre CEEA y otros EAN, como nerviosismo, aburrimiento, cansancio, soledad o sentirse demasiado ocupado. Sin embargo, la influencia de los EAN en el consumo de alcohol ha sido definida en otras investigaciones en población adolescente catalana. Un ejemplo es el estudio de Ahonen et $\mathrm{al}^{32}$, que utiliza la encuesta FRESC de Barcelona y aplica un índice aditivo de 6 ítems que incluye sentirse fuera de lugar, triste o deprimido, cansado, nervioso, aburrido, desesperanzado y con dificultades para dormir. En el citado estudio, el "consumo problemático de alcohol" es definido a partir de la presencia de al menos dos criterios entre: consumo de fin de semana, beber en bares o fiestas con amigos, haber bebido 4 o más bebidas alcohólicas en la misma ocasión, uno o más episodios de borrachera en los últimos seis meses y haber comprado alcohol por sí mismos. Los ORs con respecto al consumo problemático de alcohol así definido fueron para las edades de 14, 16 y 18 años respectivamente de $3,3,1,1$ y 1,5 para los chicos y de $2,8,2,0$ y 1,1 para las chicas. En nuestra investigación preferimos no aplicar índices aditivos a los EAN, ya que los diferentes ítems no conformaban una escala o una serie de criterios diagnósticos coherente como conjunto. Asi- mismo definimos el CEEA a partir exclusivamente de dos o más borracheras en el último mes.

La influencia del estado crónico de tristeza en el CEEA de los adolescentes varones en nuestro estudio resulta congruente con la teoría de la auto-medicación del malestar, aunque no es descartable una relación bidireccional tal como indican otras investigaciones. Una pregunta pertinente en este punto es cómo se expresa este sentimiento entre las mujeres. En una explotación realizada sobre los mismos datos y oleada del PFI se observó que la tristeza crónica era un factor de riesgo del consumo diario de tabaco entre las adolescentes (OR 1,6) y no entre los varones ${ }^{37}$. De esta forma, existiría en esta muestra una respuesta alternativa (CEEA o consumo diario de tabaco) según género para resolver los problemas de tristeza.

El efecto de "sentirse presionado por los progenitores" en la probabilidad de CEEA puede adquirir, como en la tristeza crónica, un carácter bidireccional. Los y las adolescentes practicarían CEEA por sentirse presionados y podrían sentirse presionados como resultado de sus prácticas de consumo. En cualquier caso, la asociación muestra el efecto de los estilos parentales autoritarios, no de los autoritarios o centrados en la negociación y la coresponsabilización intergeneracional. Más destacada resulta la aminoración del riesgo propiciada por factores como el origen inmigrante de las familias, el control parental de la hora de llegada a casa y la comunicación con el padre entre las jóvenes.

En cuanto a la primera variable citada (origen inmigrante de los progenitores), el resultado puede entenderse como consecuencia del healthy immigrant effect, que ha sido definido para otros consu- 
mos, como el tabaco ${ }^{38}$. No obstante, las investigaciones sobre CEEA e inmigración ofrecen resultados dispares de acuerdo con los diferentes grupos étnicos y culturales analizados y sus patrones derivados de consumo o evitación de bebidas alcohóli$\operatorname{cas}^{39}$.

En relación al papel atenuante de las dos variables "control parental del horario" y "comunicación con el padre" se pueden indicar algunas hipótesis a raíz de otras explotaciones de los datos del $\mathrm{PFI}^{40}$. En principio, la implicación del padre en la vida de las adolescentes puede conformarse como un plus de mayor comunicación intrafamiliar en un contexto caracterizado todavía por modelos desiguales según género en la distribución del cuidado y educación de los hijos, esto es, en un contexto donde la madre asume regularmente esta tarea. Esta mayor calidad de la comunicación intrafamiliar está posiblemente asociada a mayores niveles de control sobre la vida de las adolescentes y a una mayor capacidad de los progenitores para ofrecer conjuntamente apoyo a las menores que están atravesando situaciones de adversidad. Por otro lado, la inexistencia de un efecto atenuante de este factor en los varones podría estar asociada a la mayor tolerancia familiar de esta práctica entre los chicos. Asimismo, la combinación de soporte social y control parental del horario de llegada a casa podría estar influyendo indirectamente en el CEEA de las adolescentes mediante la mayor supervisión del grupo de iguales de las jóvenes. Pensemos que la proclividad de la camarilla al CEEA es uno de los factores más destacados en la literatura ${ }^{41}$. Controlar el horario de llegada a casa los fines de semana es una forma obvia de limitar las probabilidades del CEEA, pues este tipo de consumo es más frecuente durante las noches de los fines de semana, pero se muestra a la vez como un indicador de un estilo parental basado en la supervisión del ocio de las adolescentes.

El análisis de los factores familiares y psicosociales asociados al CEEA en adolescentes debe considerarse una línea de investigación relevante para la prevención de los problemas de abuso/dependencia de alcohol en la vida adulta. En un país donde el CEEA en sus modalidades populares como el "botellón" es una práctica bastante enraizada en la población juvenil, las políticas preventivas no han involucrado a las familias como actores centrales. De los datos de esta investigación se deduce que tanto los estilos parentales como los EAN deberían formar parte de las estrategias preventivas comunitarias. En esta recomendación coincidimos con distintos trabajos que, en otros contextos, han evidenciado la importancia de programas formativos dirigidos a las familias con objeto de mejorar las habilidades comunicativas de los progenitores, reforzar los vínculos intergeneracionales y las capacidades de supervisión y control de los adolescentes ${ }^{42,43}$. Dicho esto, conviene subrayar que se necesitan más investigaciones, preferentemente longitudinales y mixtas (cuantitativas-cualitativas), para valorar con mayor precisión el papel de estos factores en el consumo de alcohol entre nuestros adolescentes y jóvenes.

\section{AGRADECIMIENTOS}

Queremos agradecer a Carme GómezGranell y en general a los miembros del CIIMU su disponibilidad para el desarrollo de esta investigación, así como a los adolescentes que participaron en este estudio y a sus familias. También queremos destacar las excelentes aportaciones de los evaluadores de la Revista Española de Salud Pública. 


\section{BIBLIOGRAFÍA}

1. National Institute on Alcohol Abuse and Alcoholism. Tenth special report to the US Congress on alcohol and health. Bethesda MD: National Institutes of Health; 2000.

2. Valencia-Martín JL, Galán I, Rodríguez-Artalejo F. Binge drinking in Madrid, Spain. Alcohol Clin Exp Res. 2007; 31(10):1723-30.

3. Heffernan TM. The impact of excessive alcohol use on prospective memory: a brief review. Curr Drug Abuse Rev. 2008; 1(1):36-41.

4. Squeglia LM, Schweinsburg AD, Pulido C, Tapert SF. Adolescent Binge Drinking Linked to Abnormal Spatial Working Memory Brain Activation: Differential Gender Effects. Alcohol Clin Exp Res. 2011 Jul 18. http://dx.doi.org/10.1111/j.1530-0277.2011.01527.x. poner solo el doi, no todo el enlace

5. Abbey A, Zawacki T, Buck PO, Testa M, Parks K, Norris J, Martin SE, Livingston JA, McAuslan P, Clinton AM, Kennedy CL, George WH, Davis KC, Martell J. How does alcohol contribute to sexual assault? Explanations from laboratory and survey data. Alcohol Clin Exp Res. 2002; 26(4):575-81.

6. Archie S, Zangeneh Kazemi A, Akhtar-Danesh N. Concurrent binge drinking and depression among Canadian youth: prevalence, patterns, and suicidality. Alcohol. 2011 Aug 6.

7. Khaylis A, Trockel M, Taylor CB. Binge drinking in women at risk for developing eating disorders. Int J Eat Disord. 2009; 42(5):409-14.

8. Hingson RW, Zha W, Weitzman ER. Magnitude of and trends in alcohol-related mortality and morbidity among U.S. college students ages 18-24, 1998-2005. J Stud Alcohol Drugs Suppl. 2009; (16):12-20.

9. Grant JD, Scherrer JF, Lynskey MT, Lyons MJ, Eisen SA, Tsuang MT, et al. Adolescent alcohol use is a risk factor for adult alcohol and drug dependence: evidence from a twin design. Psychol Med 2006; 36: 109-18.

10. DeWit DJ, Adlaf EM, Offord DR, Ogborne AC. Age at first alcohol use: a risk factor for the development of alcohol disorders. Am J Psychiatry. 2000; 157(5):745-50.

11. Edwards AC, Sihvola E, Korhonen T, Pulkkinen L, Moilanen I, Kaprio J, Rose RJ, Dick DM. Depressive symptoms and alcohol use are genetically and environmentally correlated across adolescence. Behav Genet. 201; 41(4):476-87.
12. Marmorstein NR. Longitudinal associations between alcohol problems and depressive symptoms: early adolescence through early adulthood. Alcohol Clin Exp Res. 2009; 33(1):49-59.

13. Andersson B, Hibell B, Beck F, Choquet M, Kokkevi A, Fotiou A, Molinaro S, et al. Alcohol and drug use among European 17-18 year old students. Data from the ESPAD project. The Swedish Council for Information on Alcohol and Other Drugs (CAN) and the Pompidou Group at the Council of Europe. Modintryckoffset AB, Stockholm; 2007. Disponible en: http://www.espad.org/sa/node.asp?node $=637 \& i d=163 \&$ s a_content_url= $\% 2$ Fplugins $\% 2$ Fliterature $\% 2$ Fview $\% 2 \mathrm{Ea}$ sp

14. Delegación del Gobierno para el Plan Nacional sobre Drogas. Informe de la encuesta domiciliaria sobre alcohol y drogas de España [Citado 7 de octubre de 2011]. Disponible en:

http://www.pnsd.msc.es/Categoria2/observa/pdf/InformeEdades2007-2008.pdf

15. Delegación del Gobierno para el Plan Nacional sobre Drogas. Informe de la Encuesta estatal sobre uso de drogas en estudiantes de enseñanzas secundarias (ESTUDES), 1994-2008 [Citado 7 de octubre de 2011]. Disponible en:

http://www.pnsd.msc.es/Categoria2/observa/pdf/Estudes2008.pdf

16. Mota N, Alvarez-Gil R, Corral M, Rodríguez Holguín S, Parada M, Crego A, Caamaño-Isorna F, Cadaveira F. Risky alcohol use and heavy episodic drinking among Spanish University students: a two-year followup. Gac Sanit. 2010;24(5):372-7.

17. Nelson DE, Naimi TS, Brewer RD, Nelson HA. State alcohol-use estimates among youth and adults, 1993-2005. Am J Prev Med. 2009; 36(3):218-24.

18. Goldman D, Oroszi G, O'Malley S, Anton R. COMBINE genetics study: the pharmacogenetics of alcoholism treatment response: genes and mechanisms. J Stud Alcohol Suppl. 2005;(15):56-64.

19. Courtney KE, Polich J. Binge drinking in young adults: Data, definitions, and determinants. Psychol Bull. 2009; 135(1):142-56.

20. Holstein SE, Spanos M, Hodge CW. Adolescent C57BL/6J Mice Show Elevated Alcohol Intake, but Reduced Taste Aversion, as Compared to Adult Mice: A Potential Behavioral Mechanism for Binge Drinking. Alcohol Clin Exp Res. 2011; 16. http://dx.doi.org/ 10.1111/j.1530-0277.2011.01528.x.

21. Herman AI, Philbeck JW, Vasilopoulos NL, Depetrillo PB. Serotonin transporter promoter polymorphism and differences in alcohol consumption beha- 
viour in a college student population. Alcohol Alcohol. 2003; 38(5):446-9.

22. Sanchez ZM, Martins SS, Opaleye ES, Moura YG, Locatelli DP, Noto AR. Social factors associated to binge drinking: a cross-sectional survey among Brazilian students in private high schools. BMC Public Health. 2011; 31;11:201.

23. Adams M, Effertz T. Effective prevention against risky underage drinking--the need for higher excise taxes on alcoholic beverages in Germany. Alcohol Alcohol. 2010; 45(4):387-94.

24. Lonczak HS, Huang B, Catalano RF, Hawkins JD, Hill KG, Abbott RD, Ryan JA, Kosterman R. The social predictors of adolescent alcohol misuse: a test of the social development model. J Stud Alcohol. 2001; 62(2):179-89.

25. 17. Ledoux S, Miller P, Choquet M, Plant M. Family structure, parent-child relationships, and alcohol and other drug use among teenagers in France and the United Kingdom. Alcohol Alcohol. 2002; 37(1):52-60.

26. Bjarnason T, Andersson B, Choquet M, Elekes Z, Morgan M, Rapinett G. Alcohol

culture, family structure and adolescent alcohol use: multilevel modeling of frequency of heavy drinking among 15-16 year old students in 11 European countries. J Stud Alcohol. 2003; 64(2):200-8.

27. van der Vorst H, Engels RC, Meeus W, Deković M. The impact of alcohol-specific rules, parental norms about early drinking and parental alcohol use on adolescents' drinking behavior. J Child Psychol Psychiatry. 2006; 47(12):1299-1306.

28.Oostveen T, Knibbe R, de Vries H. Social influences on young adults' alcohol consumption: norms, modeling, pressure, socializing, and conformity. Addict Behav. 1996; 21(2):187-97.

29. Llorens N, Barrio G, Sánchez A, Suelves JM; ESTUDES Working Group. Effects of socialization and family factors on adolescent excessive drinking in Spain. Prev Sci. 2011; 12(2):150-61.

30. March Cerdá JC, Prieto Rodríguez MA, Danet A, Ruiz Azarola A, García Toyos N, Ruiz Román P. Parental stance towards alcohol consumption in 12- to 17 -year-old adolescents from six urban areas in Spain. Gac Sanit. 2010; 24(1):53-8.

31. Calafat Far A. Alcohol abuse by young people in Spain. Adicciones. 2007; 19(3):217-23.
32. Ahonen EQ, Nebot M, Giménez E. Negative mood states and related factors in a sample of adolescent secondary-school students in Barcelona (Spain). Gac Sanit. 2007; 21:43-52.

33. Pietraszek MH, Urano T, Sumioshi K, Serizawa K, Takahashi S, Takada Y, Takada A. Alcohol-induced depression: involvement of serotonin. Alcohol Alcohol. 1991; 26(2):155-9.

34. Nation M, Heflinger CA. Risk factors for serious alcohol and drug use: the role of psychosocial variables in predicting the frequency of substance use among adolescents. Am J Drug Alcohol Abuse. 2006; 32(3):415-33.

35. Gómez-Granell, C. El Panel de Famílies i Infància: un instrument per a la planificació i avaluació de polítiques públiques. Disponible en: http://www.ciimu.org/images/stories/CIIMU/Estudios_e I $n$ v e s t i g a c i o n e s/ 2 p a n e 1 fam/14presentacio_cggranell.pdf

36. Parada M, Corral M, Caamaño-Isorna F, Mota N, Crego A, Rodríguez Holguín S, Cadaveira F. Definition of adolescent binge drinking. Adicciones.2011; 23(1):53-63.

37. Martínez-Hernáez A, Marí-Klose M, Julià A, Escapa S, Marí-Klose P, Digiacomo S. Adolescent daily smoking, negative mood-states and the role of family communication. Gac Sanit. 2012 Jan 20.

38. O'Loughlin J, Maximova K, Fraser K, et al. Does the "healthy immigrant effect" extend to smoking in immigrant children? J Adolesc Health. 2010; 46:299-301.

39. Donath C, Grässel E, Baier D, Pfeiffer C, Karagülle D, Bleich S, Hillemacher T. Alcohol consumption and binge drinking in adolescents: comparison of different migration backgrounds and rural vs. urban residence--a representative study. BMC Public Health. 2011 7;11:84.

40. Marí-Klose P, Gómez-Granell C, Brullet C, Escapa S. Temps de les famílies: anàlisi sociològica dels usos dels temps dins de les llars catalanes a partir de les dades del Pànel de Famílies i Infància. Barcelona: Generalitat de Catalunya; 2008.

41. Trucco EM, Colder CR, Wieczorek WF. Vulnerability to peer influence: a moderated mediation study of early adolescent alcohol use initiation. Addict Behav. 2011;36(7):729-36.

42. Ashery RS, Robertson EB, Kumpfer KL, eds. Drug Abuse Prevention Through Family Interventions. NIDA Research Monograph No. 177. Washington, DC: U.S. Government Printing Office; 1998. 
43. Kosterman R, Hawkins JD, Haggerty KP, Spoth R, Redmond C. Preparing for the drug free years: Sessionspecific effects of a universal parent-training intervention with rural families. J Drug Educ 2001;31(1):47-68. 